\title{
Dynamic characteristics of a multi-storey concrete building
}

H. S. WARD

\section{Mr D. A. Howells and Dr B. Nath}

Dr Ward's Paper contains a valuable discussion of the way in which foundation flexibility may affect the frequencies and mode shapes of lateral vibration of a multistorey building. It would be interesting to carry this further, and to consider values of foundation flexibility which may be met in reality and how these may be determined more accurately than hitherto possible by using numerical methods, assuming of course that elastic constants of the soil are known sufficiently accurately.

66. In the commonly accepted simplification of considering the structure as a two dimensional problem in relation to each of two axes and suppressing the motion along the third dimension, there are two degrees of foundation freedom for each plan axis, namely translation and rocking. These cause two very different stress patterns in the soil and in extreme cases of, for example, stratification it may be desirable to use different elastic constants of the soil for each type of motion. In many cases, however, other sources of inaccuracy may mask lack of accuracy in the choice of elastic constants, so that overall values can be used for both types of motion. In most cases it would be reasonable to assume a value of Poisson's ratio based on an inspection of the cores taken during the site investigation. A wave velocity determination will then enable the Young's modulus to be calculated, though at the high values of Poisson's ratio usually encountered in soil dynamics it may eventually be found more convenient to express formulae in terms of one of the other elastic constants.

67. The evaluation of the spring constants and 'equivalent mass' of soil for a material of known elastic constants is the next step. Analytical solutions to the equations of motion have been found for certain boundary conditions, but more realistic examples call for numerical methods of analysis to account for the embedment of basement storeys and, where required, plan shape of the building. For motion along the short axis of a long building a two dimensional analysis using finite differences leads to a solution for the dynamic behaviour of the soil. ${ }^{8}$ Briefly, in the case of an embedded rigid foundation block, for example, Lamé's equations of motion can be approximated by their finite difference counterparts, in which the two components of linear displacement are the unknowns to be determined. When relevant boundary conditions are introduced in these equations, including for example that during vertical translation, all points on the block have the same displacement at a given time and the whole problem reduces to finding these displacement components at the pivotal points, for a given frequency of motion. The problem is now easily solved for steadystate conditions of motion, since the original equations reduce to a set of simultaneous equations with components of displacement as unknowns. Once these components at the pivots have been determined, contact pressures under the foundation at a given frequency and subsequently the spring constant can be determined. The computer program can as readily tackle the case of rocking motion.

68. The spring constants, both for translation and rocking, are found to be functions of the exciting frequencies and the method of finite differences outlined above makes it possible to determine this relationship. A more realistic and logical assessment of problems such as that in Dr Ward's Paper would result from the evaluation of frequency dependent spring constants obtained by means of numerical procedures.

69. For multistorey structures foundation flexibility should not be allowed to have

Paper published: Proc. Instn civ. Engrs, 1969, 43 (Aug.) 553-72. 
a significant effect on the dynamic properties of the structure. If foundation flexibility lowers the fundamental frequency by more than $10 \%$ from that calculated for a rigid base it would be wise to reconsider the whole project. This sort of analysis will give valuable guidance to the structural designer even when the elastic constants of the foundation material are not known with great precision.

\section{Dr H. S. Ward}

The Author is essentially in agreement with everything Mr Howells and Dr Nath say. As a consequence of trying over a period of five years to correlate experimentally determined dynamic characteristics of tall buildings with theoretical predictions, it became apparent that the factor about which there was least information was the effect of foundation flexibility or more precisely foundation impedance. Nevertheless the simple models used in the Paper to represent foundation impedance were not intended to represent the most realistic situation possible, but merely to see if the inclusion of the extra degree of freedom might account for the relatively poor agreement between the ratios of the measured frequencies and the theoretical values obtained when a rigid base was assumed.

71. It is important of course to develop theoretical techniques that account for ground structure interaction, such as that outlined by $\mathrm{Mr}$ Howells and $\mathrm{Dr}$ Nath. If these theoretical methods are to reflect truly the performance of actual foundation systems, however, they must incorporate information obtained from experimental observations of such systems. This input of experimental evidence is essential since with our present knowledge of soil mechanics it is unrealistic to predict the dynamic properties of soils from theoretical considerations.

72. Although experimental work in this area dates back to the Degebo ${ }^{1 \theta}$ study in 1933, there is still a need for careful experimental work because there are many pitfalls for the unwary. With this view in mind the Division of Building Research of NRC are embarking on an experimental programme which will investigate the dynamic impedances of small sized foundation elements (plan dimensions of the order of $10 \mathrm{ft}$ ). These measurements will be correlated with such factors as soil profiles and soil strength properties obtained from core samples. The information will also be used to see if it is possible to extrapolate to the dynamic behaviour of actual building foundation systems.

73. It is difficult to be explicit about how much one should allow the foundation flexibility to lower the fundamental frequency of multistorey structures. It might be argued that a $10 \%$ reduction from that calculated for a rigid base could be an indication of poor soil strength. On the other hand there are instances in North America where economic factors have encouraged the use of raft foundations rather than piles as the means of supporting multistorey structures on sensitive leda clays. It is conceivable that this solution might lead to more than a $10 \%$ reduction in fundamental frequency. In view of our limited knowledge in the area of the dynamic behaviour of foundation systems, however, the figure of a $10 \%$ reduction might serve as a limit beyond which more caution should be exercised.

\section{References}

18. NATH B. Effect of embedment on static and dynamic contact pressures under a strip footing. (To be published.)

19. Hertwig A. et al. Die Ermittlung der für das Bauwesen wichtigsten Eigenschaften des Bodens durch erzwungene Schwingungen. Springer, Berlin, 1933. 